\title{
ORGANIZACIONES REGIONALES Y SUPRANACIONALIDAD: UNA SOCIOLOGÍA POLÍTICA DE LA INTEGRACIÓN CENTROAMERICANA
}

\section{REGIONAL ORGANIZATIONS AND SUPRANATIONALISM: A POLITICAL SOCIOLOGY OF THE CENTRAL AMERICAN INTEGRATION}

Kevin Parthenay*

\section{RESUMEN}

El artículo se inscribe en el marco de un estudio global de la evolución institucional del Sistema de Integración Centroamericana (SICA). Se analiza la transformación de las funciones y actitudes de los actores regionales y, en perspectiva, la progresiva constitución de una función pública supranacional en Centroamérica. Este trabajo de Sociología Política se funda sobre una investigación de campo, revisión de archivos y más de 60 entrevistas.

PALABRAS CLAVE: AMÉRICA CENTRAL * INTEGRACIÓN REGIONAL * SOCIOLOGÍA POLÍTICA * CAPITAL SOCIAL * GESTIÓN

\section{ABSTRACT}

This article is part of a general study of the institutional evolution of the Central American Integration System (SICA). It's analyzed the changing roles and attitudes of regional actors and, in such a perspective, the progressive establishment of a supranational civil service in Central America. This paper of political sociology is based on field work, analysis of archives and more than 60 interviews.

KEYWORDS: CENTRAL AMERICA * REGIONAL INTEGRATION * POLITICAL SOCIOLOGY * SOCIAL CAPITAL * MANAGEMENT

\section{INTRODUCCIÓN}

El objetivo del artículo es entender e identificar los factores de cambio dentro del Sistema de la Integración Centroamericana (sICA), de lo cual se puede observar que los aspectos formales de cambio son más limitados que sus dinámicas reales; es decir, "lo que ves no es siempre lo que obtienes” (Gardini 2011). A veces, la práctica se distancia de la legalidad formal. Centrándose en el cambio institucional endógeno, se puede mencionar cuatro tipos posibles de cambio; el primero remite a la crisis de paradigma generada

Center for International Studies and Research (CERI) at Sciences Po, Paris, Francia.

kevin.parthenay@sciencespo.fr 
por el cuestionamiento de las bases cognitivas, debido a una acumulación de anomalías (Hall 1993); el segundo se refiere a la idea de "equilibrio roto", producto de un cambio en la representación dominante asociada con la política (True, Jones y Baumgartner 2006); el tercero es un tipo de cambio gradual (Pierson 2004) y el último, el cambio de tipo"gradual transformador"(Streeck y Thelen 1999, Mahoney y Thelen 2009), que implica cambios que se producen por la acumulación progresiva de los ajustes, a menudo invisibles e insensibles. En esta perspectiva, los aspectos formales de cambio institucional no son tan relevantes. Por lo tanto, se quiere observar los cambios en sus dinámicas más informales y menos perceptibles. En este artículo, se demostrará que a pesar de tener un marco intergubernamental de integración regional, se han desarrollado rasgos de supranacionalismo en la práctica y el ordenamiento organizacional del sica.

\section{ENTENDER EL CAMBIO INFORMAL}

La perspectiva de esta investigación es la deconstrucción de algunos diseños vinculados al análisis del cambioy la observación del papel de los actores endógenos dentro del fenómeno organizacional.

En cuanto al cambio, se fundamenta en la"teoría del cambio gradual transformador" de James Mahoney y Kathleen Thelen (Mahoney y Thelen 2010, Bonoli y Palier 1999) que consiste en un proceso de cambio lento, produciendo reformas de gran escala por un efecto de acumulación. Por otra parte, la Sociología Política considera que el cambio puede ocurrir por el uso ordinario de las normas por los actores (Croziery Friedberg 1992) y/o el contexto político existente (Thelen y Mahoney 2009). Esta concepción del cambio que se realiza a través de acumulación de ajustes y a través de la repetición de micro-comportamientos orienta hacia una Sociología Política de la integración centroamericana (Parthenay 2013).

En este sentido, por lo tanto, se consideraal fenómeno organizacional como una "construcción". Michel Crozier y Erhard Friedberg creen que "un conjunto de reglas o de datos estructurales y jerárquicos" $(1977,59)$ no son suficientes para entender el funcionamiento de las organizaciones. De hecho, los actores estratégicos producen "efectos perversos" o "contra-intuitivos"; en otros términos, la acción humana produce su cuota de consecuencias imprevistas, impredecibles e incluso, disfuncionales. Por otra parte, muchos autores han examinado la posición y el papel del actor dentro de la organización (Crozier y Friedberg 1992, Lequesne 1996, Lagroye y Offerlé 2011). Esas cuestiones se han estudiado también desde la Antropología (Abélès y Bellier 2000).

En la perspectiva del institucionalismo sociológico, muchos autores se han interrogado sobre la autonomíade los actores dentro de la organización. Se comparte la premisa de que "una situación organizativa determinada no restringe totalmente a un actor. Todavía conserva un grado de libertad y de negociación. Con este grado de libertad, cada actor tiene capacidad de poder sobre los demás" (Crozier y Friedberg 1992, 91). Con base en lo anterior, se considera que la posición institucional no es suficiente para entender el funcionamiento o el cambio de/en la institución, ya que solo proporciona una imagen estática de esta. Las personas que figuran en las posiciones y sus perfiles, dan mayor información sobre el verdadero papel de los actores dentro de las organizaciones, en este caso, el sica. El margen de maniobra se debe a varios factores: 1) la acción individual implica una gran cantidad de consecuencias impredecibles, no deseadas y disfuncionales, el fenómeno organizacional siendo un constructo que resulta esencialmente en interacciones humanas y 2) las normas que rigen $y$ regulan el funcionamiento de la organización no existen independientemente de los actores que las utilizan.

La Sociología Política considera que las organizaciones definen un conjunto de reglas, específicamente, las posiciones institucionales, pero estas no existen independientemente de los actores que las interiorizan (Lagroye y Offerlé 2011). De hecho, se puede crear un desfase entre las acciones de los actores estratégicos y las normas vigentes, lo cual causaría consecuencias no deseadas. 
El desfase puede existir también entre el comportamiento de los actores y la posición ocupada al interior de la organización. Este fenómeno es calificado según "la relación al puesto" que es "la manera con la que los agentes perciben su posición, lo que esa posición les permite hacer o decir, lo que abre como posibilidades de carrera o como oportunidad para aumentar su bienestar y la posibilidad de apropiación de una reforma posible" (Lagroye y Offerlé 2011, 21). Esta discrepancia entre el comportamiento normal y esperado se refiere a lo que Erving Goffman conceptualizaba como la "distancia con respecto al rol" (2000). Detrás de la ocupación de una posición institucional (comportamiento requerido), se considera que todas las disposiciones de una persona hacen posible una distancia con respecto al rol real del actor (comportamiento real). Este concepto de "rol social", basado en la metáfora teatral de Goffman, se retoma con Jacques Lagroye, quien diferencia la posición institucional de un actor con las oportunidades de rol alternativo.

Frente a esa teoría, los procesos de socialización y las trayectorias profesionales son fundamentales para entender la relación entre la posición institucional y el comportamiento de los actores. El objetivo es comparar los comportamientos prescritos con la conducta real para demostrar en qué medida la distancia entre la posición y el rol ayuda a entender el cambio.

Empíricamente, esta reflexión surge de la observación de las cumbres presidenciales. A pesar de las normativas, el órgano supremo del sica, así como, los presidentes mismos, no asumen plenamente su posición institucional mediante la adopción de un rol distanciado. La principal consecuencia de este distanciamiento es el intento de monopolizar el campo del poder centroamericano (Parthenay 2013). Sin embargo, este comportamiento, ha sentado las bases de una disfuncionalidad institucional que ha favorecido la intervención de otros actores en este campo. Así, se destacan dos ideas centrales: 1) otros actores han participado en la promoción del cambio en el sica y 2) el procesode distanciamiento al rol es aplicable a otros agentes regionales que operan dentro el sICA, tal como los funcionarios.

\section{ESTUDIAR EL CAMBIO INFORMAL}

Surgen varias interrogantes de investigación que guían este capítulo: ¿cómo se puede "localizar" el cambio cuando este es lento, casi imperceptible y de carácter informal? Es una de las debilidades de la teoría del cambio institucional gradual, ya que se dificulta la observación empírica de los cambios al ser focalizado sobre la informalidad y un ritmo particular (lento, gradual y cumulativo) ¿Cómo los nuevos actores han intervenido en un campo de poder formalmente monopolizado por los presidentes centroamericanos? Y ¿según cuáles estrategias $y$ a través de qué recursos $y / 0$ competencias? ¿Cómo los agentes pueden alejarse de las normas del sica que rigen su posición institucional propia? Por último, ¿̇cómo esos actores "transgresores" reinventan sus funciones $y$ transforman su "rol" dentro de la organización?

Según Sheingate (2003), las reglas institucionales son ambiguas y equívocas, además regulan situaciones complejas; asimismo, los actores con capacidades cognitivas limitadas, las conocen de manera limitada (véase el modelo de la racionalidad limitada de Simon Hebert 1947). Con una comprensión limitada, pueden surgir diferentes interpretaciones posibles de las reglas, potencialmente conflictivas, que crean una ambigüedad institucional.

Esto demuestra la importancia de considerar no solo las normas sino también sus prácticas. En este sentido, como lo plantea la hipótesis inicial, las normas no existen independientemente de los actores que las utilizan o incorporan (Lagroye y Offerlé 2011). Por último, las reglas deben estar anteriormente aplicadas e implementadas. En consecuencia, los reglamentos pueden ser interpretados de manera diferente o tener una eficacia subóptima (ej. implementación parcial), lo cual genera también ambigüedad institucional.

La hipótesis de esta investigación se basa en este fenómeno: entre más flexibles son las normas $y / 0$ fuerte es la ambigüedad institucional, más probable es la distancia del rol frente a la posición institucional (hipótesis 1). Para trabajar esta hipótesis, es necesario identificar las normas y principalmente, la práctica de esas normas. Como se ha sugerido anteriormente, 
las capacidades cognitivas de los actores pueden tener incidencia sobre la práctica de las normas. De hecho, cuando un individuo se integra a una organización, lleva con él las herencias de sus socializaciones previas formando así un patrimonio (capital social, simbólico y/o cultural). La práctica de las normas no solo está sujeta al fenómeno de la ambigüedad institucional, sino también al propio individuo.

Teniendo en cuenta una vez más que las normas no pueden existir independientemente de los actores que las utilizan y que el poder dentro de la institución no solo depende de las posiciones ocupadas, sino de disposiciones personales, se formulala segunda hipótesis: entre más consolidadas son las disposiciones individuales (acumulación de capital y de recursos), másprobableserála distancia de rol respecto a la posición institucional (hipótesis 2). Esta hipótesis se presenta con base enuna propuesta delos sociólogos de las organizaciones, quienes combinan el poder del actor dentro de la organización a la zona de la incertidumbre que controlan. Afirman que "la segunda fuente importante de poder que se encuentran en una organización está vinculada a todas las incertidumbres que crecen en torno a la relación entre las organizaciones y su entorno" (Crozier y Friedberg 1977, 85). Esta zona gris está construida tanto en la relación que se construye con el entorno como a través de los propios recursos individuales disponibles.

\section{PROSOPOGRAFÍA Y COMPARACIÓN}

Se llevará a cabo un análisis empírico profundo de las trayectorias profesionales de los actores regionales. Desde el punto de vista cualitativo-cuantitativo, se trabaja con un análisis prosopográfico y con unas comparaciones secuenciales. En este trabajo, se observa una población circunscrita: los secretarios ejecutivos. Se contabiliza 16 secretarios ejecutivos pertenecientes a las tres secretarías estudiadas: económica, social y ambiental. Para cada uno de los individuos que componen esta población, se recopila información socio-biográficas y datos sobre la trayectoria académica y profesional. Anteriormente, se procede a una compilación de todas las normas que regulan cada posición institucional.

CUADRO 1

LOS SECRETARIOS EJECUTIVOS DEL SICA

\begin{tabular}{|c|c|c|c|}
\hline ÓRGANO & INDIVIDUO & NACIONALIDAD & MANDATO \\
\hline \multirow{6}{*}{ CCAD } & Jorge Cabrera & Guatemala & $1990-1997$ \\
\hline & Mauricio Castro & Costa Rica & 1998-2003 \\
\hline & Macro Gonzales & Salvador & 2004-2008 \\
\hline & Roberto Rodríguez & Salvador & $2008-2010$ \\
\hline & José Novelo & Belice & 2011 \\
\hline & Nelson Trejo & Honduras & 2012-2016 \\
\hline \multirow{6}{*}{ SIECA } & Rafael Rodríguez & & 1991-1994 \\
\hline & Gerardo Zepeda & Guatemala & 1994-1995 \\
\hline & Haroldo Rodas & Guatemala & 1995-2008 \\
\hline & Alfonso Pimentel & Guatemala & 2008-2009 \\
\hline & Yolanda de Gavidia & Salvador & $2009-2011$ \\
\hline & Ernesto Torres & Salvador & 2011-2015 \\
\hline \multirow{5}{*}{ SISCA } & Ramón Serna & - & 1995-1999 \\
\hline & Hugo Morgado & Panamá & $2000-2004$ \\
\hline & José Sermeño & Salvador & $2004-2007$ \\
\hline & HazelEscrich & Salvador & $2008-2012$ \\
\hline & HazelEscrich II & Salvador & 2012-2016 \\
\hline
\end{tabular}

Fuente: Elaboración propia. 
Para cada secretario, se estableció una biografía detallada sobre la base de trabajo de investigación biográfico (internet, fuentes primarias, fuentes secundarias, memorias institucionales $y$ archivos) complementado por entrevistas semi-estructuradas realizadas con el individuo o de manera cruzada con antiguos colegas y/o colaboradores. Aunque en América Central, la cultura política otorga una importancia fundamental a la persona, por la estructura radial de la sociedad, la experiencia del investigador permitió destacar el hecho de que esos individuos no se ubican con facilidad $y$ tienen poca visibilidad pública. Este hallazgo, ampliamente confirmado en el sica, produce una opacidad importante de la vida institucional. Explica así la ausencia de análisis con un enfoque sociológico desarrollado hasta la actualidad. Una de las contribuciones empíricas de este trabajo es también dar a conocer a estos actores. En el plano teórico, se contribuye a abrir las puertas a nuevos enfoques para entender la dinámica de transformación de la integración regional.

El tratamiento de estos datos llevó a movilizar una herramienta metodológica específica: la prosopografía. Los objetivos de la prosopografía son:

... crear diccionarios y bases de datos utilizadas para varios estudios, la prosopografía se trata con más frecuencia de una descripción neutral. Sin embargo, identificar a un grupo y la información relevante a su respectoya es aplicar muchas suposiciones que necesitan explicaciones (Verboven, Carlier y Dumolyn 2007, 7).

Este método se articula con las teorías sociales de Pierre Bourdieu en el que se propone "recoger datos sobre los individuos de un mismo campo... esta información deberá informar a la vez sobre los recursos relacionados con los orígenes de los individuos, sino también sobre el capital simbólico del mismo campo, la posición dentro del campo y de su posición sobre los temas claves del campo" (Bourdieu 1986).

Para identificar las principales evoluciones de rol, se compara las disposiciones de los titulares de los puestos y las situaciones de ambigüedad institucional. Esta comparación tiene como objetivo identificar las especificidades $y$ establecer comparaciones internas en cada periodo e identificar las tendencias a largo plazo. El rol descrito como gradual, lento o cumulativo, resulta difícil observarlo, sobre todo cuando se produce en las pequeñas organizaciones de manera informal o en oposición a la norma existente.

La comparación permite la validación de presupuestos teóricos. Se fundamenta sobre la perspectiva de Durkheim que hace de la comparación, la única herramienta para examinar las pruebas de la existencia de una relación causal sociológica (Hassenteufel 2005). La comparación secuencial que se hace en este trabajo pondrá a prueba las dos hipótesis para cada secuencia, ya que se puede observar para cada periodo, el estado de ambigüedad institucional, las disposiciones individuales y por último, comparar sus cambios respectivos en el tiempo.

La construcción de la comparación en base al process-tracing permite el aislamiento de los factores determinantes del cambio en el sica. El process-tracing ha sido definido recientemente por George y Bennett como "un método para identificar el proceso de intervención causal, la cadena causal y los mecanismos causales, entre una variable independiente y una o más variables dependientes" (2005, 206). Las variables independientes serán las disposiciones individuales y la ambigüedad institucional y la variable dependiente será la ampliación de los actores dentro del sica. Por lo tanto, se examinará el estado de los arreglos institucionales y las disposiciones individuales en tres momentos. La primera secuencia es el momento inicial que remite a la "primera elección", la primera selección de un individuo para encarnar una posición institucional $\left(\mathrm{T}^{=0}\right)$. Esta elección es estratégica y fundadora para la organización. La segunda secuencia corresponde a la segunda selección del personal. De hecho, a través de esta opción comparativa, se toma en cuenta la variable de aprendizaje organizacional (Crozier y Friedberg 1977) y para identificar los cambios inmediatos $\left(\mathrm{T}^{+1}\right)$. Para obtener la máxima capacidad de identificacióndel cambio en el 
largo plazo, la tercera secuencia corresponde a la última selección de personal $(\mathrm{T}=\mathrm{Z})$.

\section{INSTITUCIONALIDAD CENTROAMERICANA}

La historia institucional de los Secretarios Ejecutivos es mucho menos densa que la de los Secretarios Generales del sica, quienes disfrutan de una larga trayectoria histórica desde 1951, bajo la protección de la Organización de los Estados Centroamericanos (oDEcA).

\section{UNA PERSPECTIVA HISTÓRICA DE LAS} ORGANIZACIONES REGIONALES

La mayoría de las secretarías sectoriales tiene una vida institucional relativamente breve. De hecho, la Secretaría del sector ambiental fue creada en 1989, por el Convenio Constitutivo de la Comisión Centroamericana de Ambiente y Desarrollo (CcAD), y la Secretaría del sector social en 1995, por el Tratado de Integración Social Centroamericana (TISCA). La Secretaria General del sector económico (sG-SIECA) es una excepción; la organización anterior a esta fue creada en 1960 por el Tratado General de Integración Económica, la cual dio origen a la Secretaría Permanente de la Integración Económica.

La creación de la CcAD es anterior a la de todos los órganos de la institucionalidad del sica. La razón principal es que el "tema ambiental es un tema neutral", utilizado estratégicamente en el período de paz para recuperar el pulso de la cooperación intergubernamental. Así, en el contexto de la transición y la terminación del conflicto regional en América Central, los actores gubernamentales estaban promoviendo la reactivación de la cooperación y del diálogo mediante la creación de la Comisión Centroamericana de Ambiente y Desarrollo. En ese momento, un equipo de expertos trabajaba en la creación de esta comisión, en la cual se encontraban dos futuros secretarios ejecutivos, Jorge Cabrera y Marco Antonio Gonzales. El 30 de agosto de 1989, los representantes de Costa Rica, El Salvador, Guatemala, Honduras y Nicaragua, declaran su voluntad de elaborar una Convención Constituyente de la ccad. No fue sino hasta 1990 que el Convenio entró en vigor tras su ratificación por tres Estados: El Salvador, Honduras y
Guatemala. Al año siguiente, un protocolo de la Convención se firmó, lo que permitía la adhesión de Belice y Panamá. Durante sus primeros años estuvo ubicado en la ciudad de Guatemala, posteriormente, la sede de la ccabse trasladó a San Salvador con la unificación de las secretarías en $1997^{1}$.

La Secretaría Ejecutiva del sector social es el producto de una trayectoria caótica. Las primeras iniciativas centroamericanas de integración social se han iniciado y ejecutado por las primeras damas. En aquel momento, se había creado la Comisión Regional de Asuntos Sociales (cras), con sede en Ciudad de Panamá. El surgimiento del sector social nació de un deseo compartido de las primeras damas para compartir la extensa experiencia en este ámbito, a fin de apoyar también "socialmente" la dinámica política iniciada por el Protocolo de Tegucigalpa. Posteriormente, nació un proceso nacional de institucionalización a través del cual las primeras damas integraban el ministerio social más fuerte del país. Sin embargo, para crear el Tratado Centroamericano de Integración Social (TISCA), así como, regular y organizar el funcionamiento de las instituciones sociales, se asignó a los Ministros de Relaciones Exteriores. Este tratado permitió la creación de la Secretaría Ejecutiva de la SISCA (SE-SISCA) en marzo de 1995.

Para la creación de las secretarías, pocas normas precisaron la posición institucional de los nuevos secretarios que se movían entonces en un cierto vacío normativo. Cuando existen estas normas, se destaca sobre todo su grado de imprecisión. Además, entre las mismas secretarías, el marco normativo era variable, lo que no dejaba de generar una confusión en las relaciones entre órganos y funcionarios.

\section{DESESTABILIZACIÓN ORGANIZACIONAL}

El 12 de diciembre de 1997nacela reforma institucional; no obstante, con esta los Secretarios ejecutivos fueron sumergidos en una ambigüedad institucional masiva. El objetivo de

1 En 2014, se replanteó el debate sobre regresar la sede a la Ciudad de Guatemala. 
racionalización y modernización de esta reforma implicaba, entre sus principales medidas, la unificación de las secretarías sectoriales para crear en San Salvador, una "Bruselas Centroamericana” (Sánchez 2009).

La unificación de las secretarías iniciada en 1997, generó su cuota de consecuencias no deseadas en el sica, las cuales se explican por el carácter incompleto de la reforma. En aquel momento, Guatemala y Costa Rica demostraron un fuerte nacionalismo, simbolizado por una fuerte resistencia a la centralización y a la transferencia de las secretarías de su territorio.

La sede de la siecase mantuvoen la ciudad de Guatemala, ya que su desarrollo institucional $y$ las raíces históricas nacionales fueron factores que permitieron explicar esta resistencia. También hay que mencionar los casos de los Secretarios del Consejo de Educación y Cultura Centroamericano (cecc), del Mercado Común Centroamericano (мсса) y del Consejo de Agricultura Centroamericano (cac) cuyos asientos también se mantuvieron en Costa Rica.

En última instancia, solo son tres secretarías que se han transferido a San Salvador: la SIcA, la ccady la $\operatorname{sitca}^{2}$ (Turismo). Además, para algunas secretarías, la transferencia fue incompleta debido a solicitudes nacionales, como lo fue el caso de la ccad, en el cual el traslado a San Salvador se hizo solo para la parte "gestión de proyectos" y el departamento de "gestión administrativa $y$ financiera" se mantuvo hasta el año 2011, en Guatemala.

El carácter incompleto de las reformas legales genera ambigüedades institucionales. De hecho, los presidentes unificaron las secretarías sin preocuparse de definir las reglas institucionales necesarias para estructurar las relaciones inter-organizacionales. De este modo, la unificación era una "centralización"con la omisión del marco normativo adecuado. Por esta razón, los secretarios ejecutivos, al llegar a San Salvador, se enfrentaron a un contexto ambiguo de interacción frente al Secretario General, que no dejó de generar conflictos abiertos.

2 Cuya sede estaba anteriormente en Managua, Nicaragua.
Por otra parte, la unificación se llevó a cabo sin la elaboración de un Tratado Único, lo cual se había previsto inicialmente. La consecuencia directa para el sica fue un funcionamiento organizacional sin marco administrativo ni reglamentario. A pesar del activismo de los actores regionales para poner en práctica esta unificación, la ineficacia del presidencialismo colectivo y los desastres naturales provocados por el Huracán Mitch (noviembre de 1998) obstaculizaron la implementación de la reforma. En el periodo posterior, la producción normativa, así como, el compromiso general vis-à-vis de la integración centroamericana se redujeron.

En resumen, la reforma de 1997 no fue decisiva para los cambios directos generados,sino por los vacíos legales, las distorsiones jurídico-organizacionales y todas las consecuencias no deseadas que crearon un entorno de ambigüedad institucional fuerte en el sica. Estos fenómenos han llevado a la apertura de varias ventanas de oportunidad para los funcionarios del sicaque les permitieron redefinir sus roles dentro de la institución.

\section{INSTRUMENTALIZACIÓN DE LAS NORMAS}

La ambigüedad de las normas ha creado una zona de incertidumbre acerca de la posición institucional de los secretarios. De hecho, se observó que pocas normas precisan las posiciones institucionales o las relaciones jerárquicas existentes entre esos altos funcionarios del sica. En efecto, si la relación jerárquica vigente entre el Secretario Ejecutivo y el Consejo de Ministro de tutela se define por un tratado, los secretarios generales del sica han interpretado de forma distinta la relación existente con los secretarios ejecutivos. La Secretaría General acoge en su edificio sede, a las secretarias sectoriales para facilitar la prestación de servicios. La relación administrativa se rige entonces formalmente por "acuerdos de cooperación funcional". Sin embargo, algunos secretarios generales han tenido una interpretación extensiva de su relación con los secretarios. Los secretarios ejecutivos pueden tener igualmente una interpretación extensiva de su autonomía respecto al SG-SICA. 
La consecuencia de estas interpretaciones divergentes sobre la naturaleza de las interacciones $y$ las relaciones jerárquicas, $y$ por lo tanto, el papel dentro de la institución, es la conflictividad. Esta ambigüedad de rol produce tensiones importantes que a veces han dado lugar a estrategias de exit (Hirschman 1983), como ocurrió con el Secretario ejecutivo de la CCAD, Mauricio Castro (2000-2004). Si estas estrategias de exit cumplen con una interpretación extensiva de la autonomía de la secretaría y del rol de Secretario Ejecutivo en el sica, algunos SG-sica han querido que las secretarías sectoriales se vuelvan "direcciones generales sectoriales" bajo su autoridad, como fue particularmente el caso de Óscar Santamaría (20002004) y Aníbal Quiñonez (2004-2008). De esta manera, se desarrolló una "expectativa de rol" complementaria por parte del Secretario General identificable por la elaboración de nuevas reglas internas y el aumento de las relaciones conflictivas.

Para el sector social, un "acuerdo marco de cooperación funcional", fue firmado por el Secretario General del sica y el Presidente Pro Tempore de la sisca (Ministro de la PPT-cis $\left.^{3}\right)$. El acuerdo estipula que el Secretario "tiene la obligación de coordinar con la sG-sica para cumplir con los mandatos y objetivos de la cis". Esta disposición es contraria al Tratado de Integración Social (TіscA) que establece que el SIsca se vinculará a la SG-SICA, pero nunca le da obligación de coordinarse con esta para la ejecución de normas emitidas por los órganos del Subsistema Social (según el art. 14 de tisca). Además de las normas vinculantes en relación con las obligaciones de rendición de cuentas relativas a los servicios (administrativos y financieros de la sICa), esta disposición constituye una injerencia de la Secretaría General en las competencias del sisca en el sector social. Sin embargo, en 2011, la Secretaría social firmó un acuerdo de sub-sede y recibió la persona-

3 Presidente Pro Tempore del Consejo de Integración Social. lidad jurídica internacional por el Gobierno de El Salvador, terminando así con esa ambigüedad institucional.

Para el sector ambiental, el acuerdo de cooperación funcionalrevela de la misma manera una confusión de roles. De hecho, la tercera disposición del Acuerdo establece que "la Secretaría General..., va a mantener el funcionamiento de una Dirección General de Medio Ambiente (DGma) dentro de la estructura organizativa y normativa de la Secretaría General". A pesar de la unificación de 1997, el organismo dedicado a la gestión del sector ambiental está acompañado paralelamente por una Dirección General de la sG-sica. Surge aquí la cuestión de la relación entre la DGMa y la ccad. El acuerdo estipula que "el Director tendrá la función principal de apoyar y prestar servicios a la Secretaría de la ccaD y otros foros del sector del medio ambiente". En cuanto a la posición institucional, el Director de la Dgmatiene funciones distintas a las del Secretario Ejecutivo del Convenio Constitutivo de la CCAD, los cuales se definen en 1989. El objetivo es poner la posición de la SE-ccadbajo la autoridad del Secretario General. Todas estas disposiciones producen una acumulación normativaque daña el funcionamiento y la estabilidad de las instituciones a través de expectativas distintas de rol.

\section{CAMBIO ORGANIZACIONAL Y FUNCIONARIOS REGIONALES}

Para entender la construcción de una ambigüedad de rol, se analizan las distintas disposiciones individuales de los secretarios ejecutivos. La muestra $(n=15)$ permite analizar con precisión las trayectorias socio-profesionales de los funcionarios regionales y así entender los recursos de legitimidad y competencias.

\section{EXPERTOS REGIONALES}

Los individuos que han tenido la oportunidad de ocupar el cargo de secretario ejecutivo en el sica tienen un alto nivel de formación académica. 
CUADRO 2

FORMACIÓN ACADÉMICA DE LOS SECRETARIOS

\begin{tabular}{lllllllllc}
\hline & & BA $^{4}$ & LIC & MA & DOC & ING & D.PRO $^{5}$ & DIP.ND $^{6}$ & TOTAL \\
\hline \multirow{4}{*}{ CCAD } & Jorge Cabrera & - & 1 & - & - & - & - & 1 & 2 \\
& Mauricio Castro & - & 1 & - & - & 1 & 1 & - & 3 \\
& Marco González & - & 1 & 1 & 1 & - & 1 & - & 4 \\
& Roberto Rodriguez & - & 1 & 2 & 1 & - & - & - & 4 \\
& José Novelo & 1 & - & 1 & - & - & - & - & 2 \\
& Nelson Trejo & - & - & - & - & - & - & - & 0 \\
\hline \multirow{6}{*}{ SIECA } & Rafael Rodriguez & - & 1 & - & - & - & - & 1 & 2 \\
& Gerardo Zepeda & - & & 2 & - & 1 & 1 & - & 4 \\
& Haroldo Rodas & - & 1 & 1 & - & - & - & - & 2 \\
& Alfonso Pimentel & - & 1 & - & - & - & 1 & 1 & 3 \\
& Yolanda de Gavidia & - & 1 & - & - & - & 1 & 2 & 4 \\
& Ernesto Torres & - & 1 & 1 & - & - & - & - & 2 \\
\hline \multirow{3}{*}{ SISCA } & RamónSerna & - & - & - & - & - & - & - & - \\
& Hugo Morgado & - & - & - & - & - & - & - & - \\
& José Sermeno & - & 2 & 1 & 1 & - & - & - & 4 \\
& HazelEscrich & - & 1 & 1 & - & - & - & - & 2 \\
\hline \multirow{2}{*}{ TOTAL } & & 1 & 12 & 10 & 3 & 2 & 5 & 5 & 36 \\
\hline
\end{tabular}

Fuente: Elaboración propia.

Para ilustrar esta afirmación se concentra sobre el nivel de educación y el tipo de formación recibida. Con la muestra de 13 secretarios (no aparece información sobre dos secretarios), se contabilizan 13 licenciaturas, 10 maestrías, dos grados de ingeniería, tres doctorados, seis diplomados profesionales y cinco grados "no determinado" (diplomados, grados profesionales, certificados). Se recopilan 40 grados obtenidos para un total de 13 individuos, lo que representa una proporción de 3,1 grados por individuo.

Dentro de la sieca, los secretarios se caracterizan por una gran especialización en el sector económico, pero con un nivel medio de titulación. En comparación con otras secretarías, se multiplican las formaciones profesionales. El rasgo distintivo de la secretaría social es más una especialización transversal en lo

$4 \quad$ BA: Bachillerato. LIC: Licenciatura. MA: Maestría. DOC: Doctorado. ING: Ingeniería. social, la cual es asumida por un experto en demografía y un experto en desarrollo local. Por último, para la CCAD, los secretarios se destacan por niveles muy altos de calificación. Representan la mitad de las maestrías (5/10) y dos tercios de los doctorados obtenidos.

Para dar fuerza a esta observación empírica, es necesario un análisis comparativo. Respecto al grupo desecretarios generales, se puede ver que el nivel de las calificaciones obtenidas es bastante similar, ambas se caracterizan por un alto nivel académico. Lo que diferencia a los secretarios ejecutivos es la especialización sectorial fuerte. Mientras que los secretarios generales son generalistas con diplomas de derecho internacional, relaciones internacionales o ciencias jurídicas y sociales; los secretarios ejecutivos adquieren una fuerte

\footnotetext{
5 D.PRO: Diplomado profesional.
}

6 DIP.ND: Diplomado desconocido. 
especialización en el área que deben gestionar a nivel regional.

Lógicamente existe una fuerte especialización económica en la SIECA, sobre temas ambientales en la ccAD y sobre temas sociales (población local y el desarrollo) en la sisca. En la sieca, Rafael Rodríguez y Haroldo Rodas se especializan en la economía internacional, Gerardo Zepeda se focaliza en economía industrial y gestión de inversiones, y Yolanda de Gavidia sobre la regulación y las políticas estructurales. Por otra parte, Gerardo Zepeda, Yolanda de Gavidia y Ernesto Torres Chico tienenuna formación en Administración de Empresas, lo que tiene un interés particular respecto a los esfuerzos de reestructuración organizacional y de gestión que han impulsado en la siecadurante sus mandatos.

Para el sector ambiental, todos los secretarios recibieron una formación inicial generalista, la cual fue complementada por una estricta especialización en temas ambientales. Después de una carrera de arquitecto, el guatemalteco Jorge Cabrera se especializó en temas de ecología y de evaluación de impacto ambiental. El ingeniero costarricense, Mauricio Castro, se especializó, en la gestión de los asuntos marítimos,después de estudiar Ciencia Política e Ingeniería Civil. Marco González, un abogado especializado en derecho internacional, se formó específicamente en el derecho ambiental internacional y comparado. La especialización más fuerte pertenece a Roberto Rodríguez, quien además de estudios en Antropología social y ciencias sociales, se especializó en París en Sociología, política de desarrollo rural y legislación ambiental. Con formación estrictamente académica, se encuentra José Evelio, quien luego de una Licenciatura se especializó en Entomología.

La observación de la trayectoria académicatiene un interés descriptivo por la capacidad de diferenciación de los grupos. De hecho, se puede llamar un "técnico"al que tiene el "knowhow adquirido y desarrollado a través del aprendizaje" (Meynaud 1964, 23). Meynaud también ofrece una definición más amplia: ...la noción más aceptada de forma natural del técnico es el especialista que, por su formación o experiencia, está íntimamente familiarizado con un área o un tema. Este es el hombre que, a partir de la posesión o la parecida posesión de una competencia en un sector determinado, parece capaz de sugerir formas de resolver el problema en cuestión. El rasgo característico de los especialistas es, en principio, limitado hasta el punto que lleva su conocimiento: lo que se acerca de la posición que la práctica común denomina el experto(1964, 28).

Así, se consideran como sinónimos los términos "experto", "especialista" o "técnico". A través de su formación, los secretarios de la sIEca pueden ser considerados como "técnicos de la economía" y de sectores sociales y ambientales. Sin embargo, Meynaud especifica que de la formación sola no nace necesariamente el especialista. Hace falta también considerar su experiencia y su trayectoria profesional, la cual permite la adquisición de nuevos conocimientos, competencias relacionadas con el sector y la capacidad operativa para resolver los problemas. Así que para ver el surgimiento de un "cuerpo de expertos", es preciso observar las carreras de los secretarios ejecutivos.

\section{ALTOS FUNCIONARIOS REGIONALES}

En esta sección, se analizan las trayectorias profesionales de los secretarios ejecutivos a través de tres dimensiones: la experiencia en el Alto Servicio Civil (нғр), la experiencia gubernamental (EG) y la experiencia en la Administración Pública Internacional (EFI) ${ }^{7}$.

$7 \quad$ Para los altos funcionarios públicos, se toma también en cuenta las posiciones no gubernamentales que participan en la regulación estatal (Banco Central, instituciones públicas, etc.). Los acrónimos hacen referencia a las categorías francesas, por ejemplo, HFP para "Haute Fonction Publique" (alta función pública). Se conservan así los acrónimos para los cuadros de datos. 
CUADRO 3

LAS TRAYECTORIAS PROFESIONALES DE LOS SECRETARIOS DEL SICA

\begin{tabular}{llcccc}
\hline ÓRGANOS & INDIVIDUOS & $\mathrm{EG}^{8}$ & $\mathrm{EFI}^{9}$ & $\mathrm{HFP}^{10}$ & TOTAL \\
\hline \multirow{4}{*}{ CCAD } & Jorge Cabrera & 1 & - & 1 & 2 \\
& Mauricio Castro & - & 1 & 1 & 2 \\
& Marco González & 1 & 1 & - & 2 \\
& Roberto Rodriguez & - & 1 & - & 1 \\
& José Novelo & 1 & 1 & - & 2 \\
& Nelson Trejo & - & - & - & 1 \\
\hline \multirow{6}{*}{ SIECA } & Rafael Rodriguez & - & - & 1 & 2 \\
& Gerardo Zepeda & 1 & 1 & - & 2 \\
& Haroldo Rodas & 1 & 1 & - & 2 \\
& Alfonso Pimentel & 1 & 1 & - & 2 \\
& Yolanda de Gavidia & 2 & - & - & 2 \\
\hline \multirow{3}{*}{ SISCA } & Ernesto Torres & 1 & 1 & - & - \\
& RamónSerna & - & - & - & 1 \\
& Hugo Morgado & - & - & - & 2 \\
\hline TOTAL & José Sermeno & - & 1 & - & 23 \\
\hline
\end{tabular}

La experiencia de gobierno se entiende como el ejercicio de responsabilidades, a diferentes niveles y puestos, dentro de un gobierno o un ministerio soberano. La experiencia en la administración pública internacional es el ejercicio de funciones oficiales dentro de organizaciones internacionales $y / 0$ regionales. El cuadro 3 indica la posición más alta ocupada en cada categoría. De hecho, la obtención de esta posición en sí es el producto de una ascensión profesional lenta que está lógicamente acompañada por una mayor especialización en el sector.

Al medir la longitud de la carrera, es decir, el número de puestos necesarios para alcanzar el cargo de secretario ejecutivo, se puede medir el nivel de prestigio asociado con esta función. Como se ha señalado para los secretarios generales del sica, un desfase existe entre los perfiles de las personas contratadas $y$ las funciones que

$\overline{8} \quad$ EG: Experiencia gubernamental (ministro o viceministro).

$9 \quad$ EFI: experiencia de funcionario internacionaL. se derivan de la posición institucional. Estas indicaciones prosopográficas dan informaciones valiosas sobre el valor social de estas posiciones institucionales. Tomando en cuenta la edad promedio de entrada en función, la edad media de los secretarios ejecutivos (51 años) y de los secretarios generales (54 años) es equivalente. Es también similar la edad media correspondiente a los puestos de la alta administración pública nacional (52 años). Así, esta posición que tiene un cierto prestigio social solo se puede lograr a través de una experiencia profesional densa y de alto nivel. Ocupar el cargo de secretario ejecutivo es en sí, la culminación de una carrera de nivel nacional y regional. Se destaca que muchos secretarios son electos tras la ocupación de cargos gubernamentales, a veces cargos ministeriales de primer orden (De Gavidia y Rodas) o después de haber ocupado muchas posiciones en la institucionalidad centroamericana (Pimentel, Torres y Sermeño).

$\overline{10}$ HFP: experiencia en el alto servicio civil (consejero, director de gabinete). 
Sin embargo, el prestigio de la función y el alto nivel de especialización no es consistente con los poderes y las competencias consentidas a esas posiciones institucionales. Este desfase se puede también medir a través de la densidad de la trayectoria profesional, medida por el número promedio de puestos ocupados por los secretarios antes de asumir el cargo y la distancia temporal entre la primera posición y el nombramiento como secretario. Se trata de nueve posiciones ocupadas aproximadamente en un promedio de casi 26 años.

En el sisca, Ana Hazel Escrich ocupó el cargo de Secretario Técnico de la Presidencia de la República para el área social. También había ocupado el cargo de Ministra representante ante el Consejo de Integración Social (crs). En la SIECA, Haroldo Rodas era experto internacional del PNud en materia de negociación comercial. Yolanda Mayora de Gavidia fue Ministra de Economía del Salvador. Gerardo Zepeda y Alfonso Pimentel fueron respectivamente Secretario General Adjunto y Director Ejecutivo de la siEcA. Por último, en la ccad, Jorge Cabrera fue secretario de la Comisión Presidencial y Representante Oficial de Guatemala en las negociaciones del alides y de la Cumbre de Río en 1992. Marco González fue Director de Programa del PNud y finalmente, Mauricio Castro, Jefe del Departamento de Banco de Desarrollo Social Centroamericano de Integración Económica (BCIE). Tanto a nivel nacional como regional, la llegada al cargo de Secretario Ejecutivo es la consagración de una larga experiencia profesional en el sector de referencia y del desarrollo de una especialización de alto nivel.

Los secretarios ejecutivos salen de una esfera de profesionales del sector. Se identificó con la observación de las trayectorias profesionales, una gran movilidad entre distintos ámbitos profesionales como el asesoramiento, el servicio civil internacional o la administración civil de alto nivel. Sin embargo, la movilidad no se realiza fuera del sector de especialización. No obstante, basándose en Meynaud, la movilidad intensa de esta "elite" puede llevar a matizar la observación de la alta "especialización": "el especialista, así definido se opone al 'generalista' (que se denomina a veces el 'técnico de las ideas generales') que está definida por una capacidad a dominar las manipulaciones parciales o sectoriales y sintetizarlas" $(1964,28)$.

¿Serán los secretarios "generalistas"? ¿Tendrán la capacidad para dominar además de los temas y las agendas, también las rivalidades $y$ conflictos entre actores $y$ niveles de acción y en última instancia, formular posiciones y orientaciones consensuales? Este concepto de generalista orienta hacia la noción de tecnócrata y el "especialista" se limita al punto al que le lleva su conocimiento. En este sentido, los requisitos de la posición institucional requieren poseer otras habilidades más allá de la especialización sectorial. Los secretarios deben de tener capacidades de gestión para supervisar los equipos, de diplomacia para manejar las negociaciones intergubernamentales $y$ deben de tener una "visión global" del sector para identificar los temas prioritarios de la agenda ¿Por lo tanto, serán los secretarios ejecutivos "tecnócratas"?

\section{INFORMALIDAD SUPRANACIONAL: DEL FUNCIONARIO AL TECNÓCRATA}

\section{EMPRENDEDORES POLÍTICOS REGIONALES}

El tema de la tecnocracia se refiere a las cuestiones de la despolitización y la confiscación de posiciones dominantes por parte de "especialistas". En otras palabras, se refiere a la aparición de especialistas que dan lugar a una especie de "gobierno de técnicos" (Meynaud 1964: 32), que privaría a los políticos tradicionales, en este caso, los Consejos de Ministros, de la capacidad de gobernar frente el desarrollo de la tecnicidad. Se plantea también el tema de la aparición de una élite a nivel regional (Genieys 2000).

Se ha destacado que la alta movilidad profesional de los secretarios les pone en contacto con la esfera del poder y contribuye a ampliar en gran medida el grado de sociabilidad. Así, los secretarios tienen habilidades específicas que les permiten superar la función de coordinación para convertirse en political entrepreneurs (Lequesne 1996). Además de ser especialistas en economía, temas sociales o ambientales, los secretarios han ocupado un número variable de responsabilidades gubernamentales directas o 
indirectas; en este sentido, tres secretarios de la CCAD han alcanzado un mandato gubernamental. Jorge Cabrera asumió el papel de Asesor para la creación de la Comisión Nacional de Medio Ambiente de Guatemala y Representante de Guatemala ante la negociación de la aLides, al igual que Mauricio Castro de Costa Rica. Por otra parte, Marco Gonzálezera Director de Política Exterior y luego, de Cooperación Internacional del Ministerio de Relaciones Exteriores de Nicaragua. José Evelio era Agente de Proyectos en el Ministerio de Agricultura y Pesca de Belice.

Los secretarios deben ser capaces de gestionar los conflictos entre los Estados, los clivajes intergubernamentales, así como, los conflictos ministeriales. Tienen que hablar el mismo idioma que los Ministros del sector y conocer los códigos del "intergovernment albargain" (Moravcsik 1993). En este sentido, las experiencias gubernamentales o ministeriales son decisivas al momento de acceder al cargo de Secretario Ejecutivo. En el caso de la sieca, la obtención de puestos de Viceministro (Rodas), Ministros (Zepeda y de Gavidia) o Asesor del Ministro y/o del Presidente de la República, también son cruciales a fin de calificar como Secretario de siECA. De hecho, pasar por el servicio civil o asumir responsabilidades gubernamentales también ayuda a medir la dimensión de prestigio.

La multiplicación de las posiciones ocupadas de Asesor presidencial, Ministro o el ejercicio de la responsabilidades gubernamentales, destaca el hecho de que las personas seleccionadas, además de ser expertos en sus campos, son profesionales ubicados a la intersección de dos campos: el de la especialización técnica y el de la política. Contratados como técnicos, los secretarios ejecutivos desempeñan con mayor frecuencia el rol de "tecnócratas", lo que no carece de crear más ambigüedad institucional en el sicA.

\section{TECNOCRACIA SUPRANACIONAL}

Se utiliza los resultados de una comparación secuencial para identificar el cambio de los perfiles seleccionados para incorporar a las secretarías sectoriales. Las secuencias se construyeron sobre la base de los elementos formales de reforma del sica, es decir, la primera reforma de 1997 y la segunda reforma de 2004.

CUADRO 4

COMPARACIÓN SECUENCIAL DE LOS SECRETARIOS EJECUTIVOS

\begin{tabular}{llll}
\hline & CCAD & SISCA & SIECA \\
\hline $\begin{array}{l}\text { SEQ1 } \\
(1991-1996)\end{array}$ & $\begin{array}{l}\text { Jorge Cabrera } \\
(1990-1997)\end{array}$ & $\begin{array}{l}\text { Ramon Serna } \\
(1995-1998)\end{array}$ & $\begin{array}{l}\text { Rafael Rodriguez } \\
(1991-1994) \\
\text { Gerardo Zepeda (1994-1995) }\end{array}$ \\
\hline $\begin{array}{l}\text { SEQ2 } \\
(1997-2003)\end{array}$ & $\begin{array}{l}\text { Mauricio Castro } \\
(1998-2003)\end{array}$ & $\begin{array}{l}\text { Hugo Morgado } \\
(1999-2004)\end{array}$ & $\begin{array}{l}\text { Haroldo Rodas } \\
\text { (1995-2008) }\end{array}$ \\
\hline $\begin{array}{l}\text { SEQ3 } \\
(2004-2007)\end{array}$ & $\begin{array}{l}\text { Marco González }(2004-2008) \\
\text { Roserto Rodríguez (2008-2010) }\end{array}$ & $\begin{array}{l}\text { José Arnoldo Sermeño } \\
(2004-2008)\end{array}$ & $\begin{array}{l}\text { Haroldo Rodas } \\
\text { (1995-2008) }\end{array}$ \\
\hline $\begin{array}{l}\text { SEQ4 } \\
(2008-2012)\end{array}$ & $\begin{array}{l}\text { Nelson Trejo } \\
\text { (2011-en curso) }\end{array}$ & $\begin{array}{l}\text { HazelEscrich } \\
(2008-2012)\end{array}$ & $\begin{array}{l}\text { Yolanda Mayora de Gavidia } \\
\text { (2008-2010) }\end{array}$ \\
\hline
\end{tabular}

Fuente: Elaboración propia. 
En la primera secuencia (1991-1996), la ambigüedad institucional no se había desarrollado. Las secretarías estaban en construcción y las relaciones jerárquicas seguían la lógica de una receta única impuesta por los ministros del sector. Además, en aquel momento, los ministros eran poco enterados de las funciones de las secretarías. Por otra parte, la evolución institucional es limitada $y$ desigual entre las secretarías. En esta primera secuencia, el contexto era de relativa estabilidad institucional con un reto predominante de consolidación. Si no se puede hablar de autonomía en esta etapa de institucionalización inicial, se identifica una correlación positiva entre el desarrollo organizacional y las disposiciones individuales.

Las disposiciones individuales de los primeros secretarios ejecutivos son desiguales $y$ relativamente débiles. De hecho, Jorge Cabrera es una excepción al tener el capital social más alto, debido en particular a su experiencia profesional que favoreció una sociabilidad amplia. Esto explica su tendencia a transgredir su rol en repetidas ocasiones sobrepasando las líneas jerárquicas y su estatus dentro de las reuniones internacionales. Este último se auto-proclamó con frecuencia "Representante presidencial" para Guatemala. A través de estas disposiciones, Cabrera ha permitido a esta institución fortalecerseen el ámbito de la institucionalidad centroamericana. Teniendo en cuenta el desarrollo institucional significativo de la SIECA, las disposiciones individuales son menos determinantes en esta perspectiva. Respecto a la SICA, permaneció en las sombras con un nivel muy bajo de actividad durante la gestión de Ramón Serna. A pesar de la estabilidad institucional, la ineficacia de los Ministros y los problemas de cartera "sociales" en el ámbito doméstico explican la ausencia de desarrollo organizacional. En resumidas cuentas, en esta primera secuencia, el estado de construcción institucional constituye una variable determinante a diferencia de la ambigüedad. Las distintas disposiciones permitieron lograr distintos niveles de desarrollo organizacional, lo que indica que esta variable es pertinente en la institucionalidad centroamericana. No obstante, no se puede hablar todavía de political entrepreneurship sino más bien de liderazgo institucional.

La segunda secuencia está marcada por la reforma de 1997 y el proyecto de unificación de las secretarías (1997-2003). Los secretarios se enfrentaron a una desestabilización institucional fuerte por el cuestionamiento de las relaciones de poder dentro del sistema. En esta configuración, se constata que las distintas disposiciones individuales fueron fundamentales para el surgimiento de un nuevo rol y de una nueva interpretación de la posición institucional. El capital social y simbólico planteado por Rodas fue decisivo al negociar la transferencia de las secretarías. Con un fuerte apoyo del gobierno, Rodas tenía la capacidad para combatir enérgicamente la propuesta de transferencia de la sieca, en San Salvador. Este capital social, cultural y simbólico le permitió tener la mayor longevidad de todos los secretarios con trece años al frente de la institución. La importancia de este capital se ilustra por la transferencia de experiencias a la secretaria social Hazel Escrich para el empoderamiento de la sisca, a partir de 2008. Rodas es también el único secretario ejecutivo quien posteriormente obtuvo un puesto ministerial (Relaciones Exteriores).

Al mismo tiempo, Mauricio Castro entró en la lucha contra el secretario general Oscar Santamaría para ganar la autonomía de la CCAD. El conflicto llevó a la salida de la ccadde la Secretaría General. Sin embargo, Mauricio Castro, a pesar de tener un capital cultural más alto que la media $(0,05 / 0,045)$, no tenía coalición de apoyo que podía soportar su lucha $y$ mantenerse al mismo tiempo dentro de la institucionalidad centroamericana, lo que explica la estrategia de salida.

El contexto de desestabilización institucional fue limitado $y$ relativo con la sisca por parte de la aceptación del secretario Morgado de una "doble sumisión jerárquica" (frente a los Ministros y al Secretario General del SICA). Se carece de información para el secretario Morgado, sin embargo, se puede deducir que su trayectoria dentro de la institucionalidad centroamericana se debe a su estrecha relación con el Ministro panameño del Consejo de la 
Integración Social ${ }^{11}$. Esta sociabilidad, junto con las deficiencias institucionales explican, en primer lugar, la incapacidad para fortalecer la institución y en segundo lugar, para reinventar un rol en el subsistema social de la integración centroamericana.

Las dos últimas secuencias (2004-2007 y 2008-2012) son las más ilustrativas para marcar la evolución de los secretarios ejecutivos de un rol de "técnico" a un rol de "tecnócrata". Como se observa, en años anteriores - con la excepción de Haroldo Rodas - se seleccionaban perfiles de técnicos para encabezar las secretarías; sin embargo, en los últimos tiempos, los perfiles seleccionados son más completos. En la sieca, los últimos dos perfiles seleccionados tienen un capital social muy alto. En 2009, fue nombradauna ex ministra de la economía salvadoreña, Yolanda Mayora de Gavidia. Después de arreglos políticos, se tuvo que nombrar a Ernesto Torres Chico en un contexto turbio. Torres tiene la mayor movilidad profesional en la muestra con un número considerable de puestos ocupados $(\mathrm{n}=27)$. También tiene una gran experiencia de la integración centroamericana y por lo tanto, un capital elevado en este campo específico como ex funcionario de la sieca y ex Director en la Secretaría General del sica.

Los últimos nombramientos dentro de la CCAD también son particularmente ilustrativos. El nombramiento del beliceño José Evelio, especialista estereotipado en materia medioambiental (entomólogo), se reasignó debido a la carga política del puesto. La renuncia de este secretario dice mucho sobre el rol cambiante de la Secretaría Ejecutiva de la ccad. Actualmente, los perfiles de técnicos no son adecuados para un trabajo que implica más que el conocimiento de un sector. Las nuevas competencias requeridas son implícitas para esta posición, la capacidad de moverse a la frontera de la política $y$ de colaborar con actores múltiples (gobiernos,

11 De hecho, como lo indican las declaraciones oficiales del Consejo de Integración Social, Hugo Morgado, antes de asumir el cargo de Secretario, fue con frecuencia representante del Ministro cisde Panamá durante las Cumbres ministeriales del sector. cooperantes y la sociedad civil). Esta transformación fue confirmada posteriormente por el nuevo Secretario, el hondureño Nelson Trejo, el perfil no solo se basa en su especialización en el sector ambiental, sino también un alto conocimiento del sector de la cooperación internacional a través de su experiencia profesional que se caracteriza por una fuerte movilidad y una alta cercanía a los cooperantes internacionales.

Por último, el desarrollo más significativo es sin lugar a dudas para el Secretario de la integración social. En este caso, la sociabilidad juega una vez más una importancia de primer orden. Ana Hazel Escrich asumió el cargo de Ministra del Consejo de Integración Social. Por otra parte, durante toda su carrera se dedicó a la integración social centroamericana, con una movilidad muy baja y un número bajo de posiciones fuera de este campo. De ahí, las buenas relaciones existentes con los ministros, que se traducen en el concepto de "binomio cIs-sIscA", permitiendo a la Secretaria de tener un rol importante tanto en la gestión de la agenda estratégica como en la puesta en marcha de las decisiones ministeriales. Esto se traduce en un fuerte protagonismo $y$ un empoderamiento gradual de la Secretaría. La SIsca obtuvo su independencia en relación con sGsica en setiembre de 2010.

La primera observación que se estableció es que la estabilidad institucional, que se entiende como una interpretación compartida y no conflictiva de las normas, es poco común en la institucionalidad centroamericana. Más allá de la reforma de 1997, que ha generado una desestabilización generalizada en la integración regional, se constata que la última ola de nombramiento de los secretarios ejecutivos fue golpeada de nuevo por una ambigüedad institucional fuerte. La ccad después de la dimisión de José Evelio se mantuvo casi dos años sin secretario. Dentro de la sIEca, el nombramiento de Ernesto Torres Chico es cuestionado por Costa Rica, la cual rechaza pagar su cuota anual a la institución. Por último y más recientemente, la reelección de Hazel Escrich es contestada por Nicaragua y Honduras, este último teniendo intención de proponer un nuevo candidato. Desde 1997, todos los secretarios evolucionaron sobre un terreno favorable a la transformación 
del rol. De hecho, al no definir claramente las posiciones institucionales, se deja espacio para interpretaciones diferentes y contradictorias.

La segunda variable basada sobre las disposiciones individuales explica ciertos fenómenos de redefinición del rol. En este caso, el capital cultural es un indicador poco pertinente para explicar el fenómeno de "distancia del rol". De hecho, se encuentra frente a una muestra compuesta por especialistas, técnicos o expertos en los respectivos sectores. Sin embargo, hay que destacar la evolución de rol de los secretarios con competencias que se sobreponen a la especialización sectorial y temática. Un factor decisivo es el capital social y simbólico que se mide a través de la trayectoria de la carrera. La movilidad profesional informa mucho sobre el volumen de capital social y simbólico acumulado por el individuo durante su carrera, permitiendo la expansión de las redes, incluyendo los lazos débiles (Granovetter 1973) y el grado la sociabilidad. Esos indicadores son decisivos a la hora de considerar el cambio. Esta variable es válida para explicar la resistencia al cambio, como se muestra en el caso de Rodas de la sIeca, pero también para explicar la promoción del cambio, como se ha sugerido en el caso opuesto de Escrich a la cabeza de la sisca. Además, se constata que las coaliciones de apoyo juegan un papel significativo en la aprehensión de cambio (Sabatier y Jenkins-Smith 1993). En esta perspectiva, se valida la hipótesis de tecnocratización supranacional por la variable relativa a las"disposiciones individuales".

\section{CONCLUSIÓN}

En respuesta a las dos hipótesis planteadas, se infiere que es a través de un contexto de ambigüedad institucional, interpretaciones contradictorias y de normas divergentes, se pudieron construir roles distantes de las posiciones institucionales formales. Por consiguiente, se concluye la importancia del contexto organizacional como un vector de flexibilización de la "relación al puesto" (Lagroye y Offerlé 2011). En segundo lugar, no se destacó una correlación directa entre las "disposiciones individuales" y la transformación de los roles; sin embargo, es importante destacar el factor de sociabilidad. Este concepto aparece en la década de 1950 por Karl Deutsch y se plantea como un factor decisivo para explicar el cambio. Usando las teorías más contemporáneas del cambio, el concepto de "coalición", como un vehículo o un obstáculo para el cambio, es también decisivo para ilustrar las transformaciones de los roles de los secretarios ejecutivos.

Aunque la distancia con respecto a la posición institucional es un factor informal de cambio, es evidente su discontinuidad, dependiendo de compromisos tácitos entre un conjunto de actores dentro del sistema. Por consecuencia, la tendencia de tecnocratización de los secretarios ejecutivos en el sica reúne necesariamente los intereses de otros actores del sistema, principalmente los ministros, que ven el potencial de apoyo técnico y la capacidad de atracción de la cooperación internacional. Por lo tanto, el uso diario de las normas contribuyeron a largo plazo a cambiar mano a mano el rol de los secretarios que de "escribientes de reuniones ministeriales" se han convertido en policy entrepreneurs que ayudan a la definición de las agendas de política pública y political entrepreneurs que participan en la transformación de las organizaciones del sica.

\section{BIBLIOGRAFÍA}

\section{LIBROS}

Baumgartner, Franck y Brian Jones. 1993. Agendas and Instability in American Politics.Chicago, EeuU: University of Chicago Press.

Bourdieu, Pierre. 2000. Propos sur le champ politique. Lyon, Francia: Presse Universitaire de Lyon.

Comisión Económica para América Latina $y$ el Caribe-Banco Interamericano de Desarrollo. 1997. La integración centroamericana y la institucionalidad regional. Santiago, Chile: Comisión Económica para América Latina-CEPAL.

Crozier, Michel y Erhard Friedberg. 1992. L'acteuret le système. Paris: Seuil.

Dabène, Olivier. 2012. "Consistency and resiliencethroughcycles of repoliticization". 
The rise of post-hegemonic regionalism. The case of Latin America. Editado por Pia Riggirozzi y Diana Tussie. London, Inglaterra: Springer.

George,Alexander y Andrew Bennett. 2005. Case Studies and Theory Development in the Social Sciences. Cambridge, EEuv: Massachusetts Institute of TechnologyMIT Press.

Hassenteufel, Patrick. 2009. Sociologie politique: l'actionpublique. Paris, Francia: ArmandColin.

Hirschman, Albert. 1983. Bonheur Privé, action publique. Paris, Francia: Fayard.

Lagroye, Jacques y Alice Lacroix. 1992. Le Président de la République: usages et genèses d'une institution. Paris, Francia: Les Presses de Sciences Po.

Lagroye, Jacques y Michel Offerlé, dir. 2011. Sociologie de l'institution. Paris, Francia: Belin.

Mahoney, James y Kathleen Thelen. 2009. Explaining institutional change, ambiguity, agency, and power. Cambridge, EEuU: Cambridge University Press.

Meynaud, Jean. 1964. La Technocratie. Mytheouréalité? Paris, Francia: Les Éditions Payot.

Onoma Ato, Kwamena. 2009 . "The contradictory potential of institutions: The rise and decline of land documentation in Kenya". Explaining institutional change, ambiguity, agency, and power. Editado por James Mahoney y Thelen, Kathleen. Cambridge, EEuU: Cambridge University Press.

Pierson Paul. 2004. Politics in Time: History, Institutions, and Social Analysis. New Jersey: Princeton Press University.

Sabatier, Paul y Hank Jenkins-Smith, eds. 1993. Policy change and learning: an advocacy coalition approach. Boulder, EEUU: Westview Press.

Sanchez, Rafael. 2009. The politics of Central American integration.New York, EEUU: Routledge.

Smith, Andy. 2010. Le gouvernement de l'Union Européenne: une sociologie politique.
Paris, Francia: Librairie Générale de Droit et de Jurisprudence-LGDJ.

Streeck, Wolfgang y Kathleen Thelen, dir. 2005. Beyond continuity. Institutional change in advanced political economies. Oxford, Inglaterra: Oxford University Press.

True, James, Bryan Jones y Frank Baumgartner. 2007. "PunctuatedEquilibriumTheory. Explaining stability and change in public policy making". Theories of the Policy Process, editado por Paul A. Sabatier. Colorado, EEUU: Westview Press.

Verboven, Koenraad, Myriam Carlier y Jan Dumolyn. 2007. "A Short Manualtothe Art of Prosopography". Prosopography Approaches and Applications: $A$ Handbook, editado por K.S.B KeatsRohan. Oxford, Inglaterra: Oxford UniversityPress.

PUBLICACIONESPERIÓDICAS

Abélès Marcy Irène Bellier. 1996. "La Commissioneuropéenne: du compromisculturel à la culture politique du compromise". Revuefrançaise de sciencepolitique 46, n. 3 : 431-456.

Bonoli, Giuliano y Bruno Palier. 1999. "Phénomènes de path dependence et réformes des systèmes de protection sociale". Revue française de science politique 49, n. ${ }^{\circ}$ : 399-420.

Bourdieu, Pierre. 1986. "L'illusion biographique". Actes de la recherche en sciences sociales 62/63: 69-72.

Gardini, Gian Luca. 2011. "Mercosur: whatyouseeisnot (always) whatyouget". European Law Journal 17, n. ${ }^{\circ}$ 5: 683-700.

Genieys, William. 2000. "Pour une sociologie comparée des élites en interaction". Revue Internationale de Politique Comparée 7, n. ${ }^{\circ}$ 2: 467-492.

Goffman, Erving. 2000. "La 'distanceaurôle' en salled'opération". Actes de la Recherche en Sciences Sociales 143, n ${ }^{\circ}$ 1: 80-87.

Granovetter, Mark, 1973. "Thestrengh of weakties". American Journal of Sociology 78, n. ${ }^{\circ}$ 6: 1360-1380. 
Hall, Peter. 1993. "Policy paradigms, social learning, and thestate. The case of economicpolicymaking in Britain". Comparative Politics 25, n. ${ }^{\circ} 3$ : 275-296.

Hassenteufel, Patrick. 2005. "De la comparaison internationale à la comparaison transnationale, les déplacements de la construction d'objets comparatifs en matière de politiques publiques". Revue Française de Science Politique 55, n. ${ }^{\circ}$ 1: 113-132.

Laitin David, GreifAvner. 2004. "A theory of endogenous institutional change". American Political Science Review 98, n. 4 : 633-652.

Lequesne, Christian. 1996. "La Commission européenne: entre autonomie et dépendance". Revue Française de Science Politique 46, n. 3 : 387-399.

Moravcsik, Andrew. 1993. "Preferences and Power in the European community: a liberal intergovernmentalistapproach". Journal of common market studies 31: 473-524.

Sheingate, Adam. 2003. "Political entrepreneurship, institutional change and american political development". Studies in American Political Development 17: 185-203.

TESIS

Parthenay, Kevin. 2013. "L’intégration régionale en Amérique Centrale. Une sociologie politique du changement". Tesis Doctoral en Ciencias políticas. Paris, Francia. Sciences Po.

OTROS

Dabène, Olivier. 2012. "Explaining Latin America's fourth wave of regionalism. Regional integration of the third kind". Ponencia pronunciada durante el xxx Congreso de la Asociación de Estudios Latino Americanos.San Francisco, EEUU, 25 mayo.

Fecha de ingreso: 26/09/2014 Fecha de aprobación: 30/01/2015 